\title{
Spatial distribution of neutral and ionic constituents in comet $\mathbf{P} /$ Halley $^{\star}$
}

\author{
R. Umbach ${ }^{1, \star \star}$, K. Jockers ${ }^{1}$, and E.H. Geyer ${ }^{2}$ \\ 1 Max-Planck-Institut für Aeronomie, Postfach 20, 37189 Katlenburg-Lindau, Germany \\ 2 Sternwarte der Universität Bonn, Observatorium Hoher List, 54550 Daun, Germany
}

Received September 6, 1996; accepted April 30, 1997

\begin{abstract}
Multislit spectra of comet P/Halley were obtained on April 10, 1986, using a focal reducer with a field of $\approx 0.5^{\circ}$ angular diameter. The number and arrangement of the slits allowed a reconstruction of the spatial distribution of principal neutral and ionic coma constituents within a frame of about $410^{5} \times 410^{5} \mathrm{~km}^{2}$ at the comet. Radial profiles and two-dimensional maps for the dust continuum, $\mathrm{CN}, \mathrm{C}_{3}, \mathrm{CH}, \mathrm{CO}^{+}$, and especially $\mathrm{CO}_{2}^{+}$, are presented in absolute intensity and column density units, respectively. Scale lengths and production rates for the radicals were determined within the Haser model. The molecular ions are not only restricted to the plasma tail but are also present in the sunward coma. Instead of forming a peak at the nucleus like the neutral radicals the ion column densities decrease rapidly in the solar direction and form a plateau extending from the nucleus into the antisolar direction. The distribution of $\mathrm{CO}_{2}^{+}$shows a stronger decrease in the tailward direction than that of $\mathrm{CO}^{+}$.
\end{abstract}

Key words: comets: individual: P/Halley multislit spectra - molecular ions - radicals spatial distribution

\section{Introduction}

To supplement the spacecraft observations of comet P/Halley 1986 III a detailed ground-based observing program was conducted at the European Southern Observatory. The observations aimed at the determination of the spatial distribution of the most interesting neutral and ionic coma constituents which show

Send offprint requests to: K. Jockers

* Based on observations at the European Southern Observatory, La Silla, Chile.

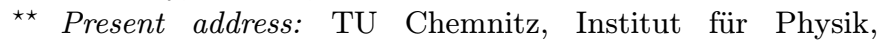
Gasentladungs- und Ionenphysik, 09107 Chemnitz, Germany. emissions in the near-UV and blue spectral ranges. Within this program the focal reducer of the Observatory Hoher List, augmented and adapted to comet observations with instrumentation provided by the Max-PlanckInstitut für Aeronomie was used at the European Southern Observatory $1 \mathrm{~m}$ telescope between March 10-16 and April 4-11, 1986. As detector an image intensifier was chosen because at the observed wavelengths it had a better sensitivity than the CCD cameras available at that time. For permanent recording photographic plates were used.

The observational data consist of direct filter images and multislit spectra. A set of direct images obtained on April 11, 1986, was analysed by Jockers et al. (1987). The present paper is concerned with the data reduction and analysis of multislit spectra taken on April 10, 1986. With the multislit technique it was possible to obtain 69 spectra, each from a different part of the cometary coma, at the same time.

The spectra show a large number of emission features due to different coma constituents. The analysed emissions originate from the molecular ions $\mathrm{CO}^{+}, \mathrm{CO}_{2}^{+}$and the neutral radicals $\mathrm{CN}, \mathrm{C}_{3}$, and $\mathrm{CH}$. Radial profiles and twodimensional maps, which describe the spatial distribution of these species and of the dust continuum, are deduced from the spectra. The spatial distribution of the neutral radicals is interpreted in terms of the Haser (1957) model. The value of this paper, in our opinion, rests in the information presented about the distribution of the cometary ions $\mathrm{CO}^{+}$and $\mathrm{CO}_{2}^{+}$. Even 10 years after the apparition of comet Halley such information has not been published elsewhere. Also the results about the dust and the neutral radicals are unique because the field of the employed instrument was well adapted to the size of the cometary coma. Comparison of the data on neutral radicals with data obtained by other authors provides confidence in our results which were obtained the oldfashioned way with an image intensifier and recorded on photographic plates. To our knowledge this is so far the only case where the multislit technique has been applied to comet observations, but 


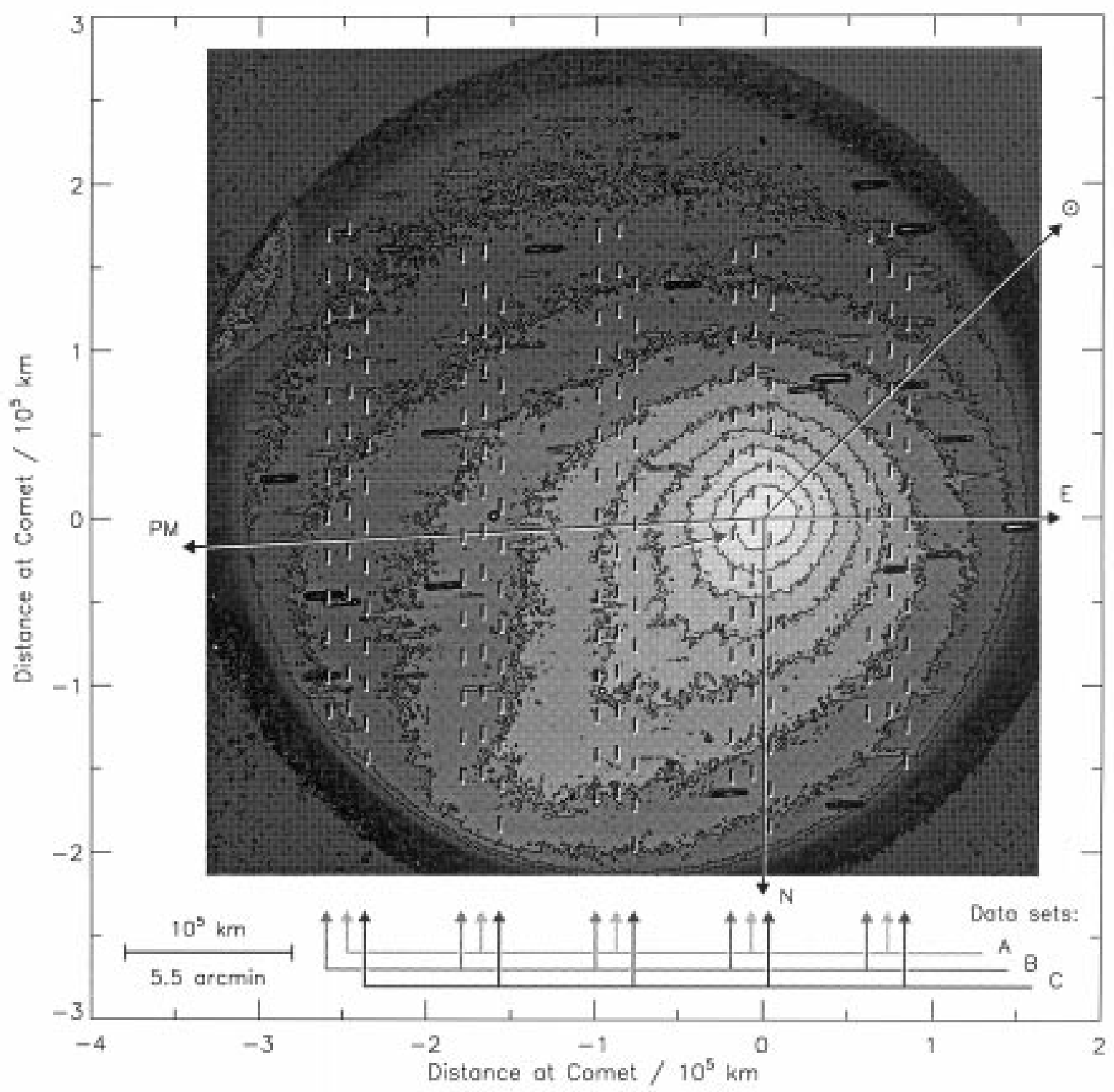

Fig. 1. Coma of $\mathrm{P} /$ Halley in the light of $\mathrm{CO}_{2}^{+}$with superimposed slit positions of the three data sets, and projected directions

the procedure is similar to the technique used in the TKS instrument flown on the Vega spacecraft (Rousselot et al. 1994; Clairemidi et al. 1990). The results of this work can be used for comparison with models of the abundance and spatial distribution of cometary constituents in comet Halley and other comets.

\section{Observations}

\subsection{Position and aspect geometry of the comet}

The mean relative position of the comet with respect to sun and earth during the observational period on April 10, 1986, was $r=1.32 \mathrm{AU}$ and $\Delta=0.42 \mathrm{AU}$, respectively; the values were computed from orbit parameters published by Green (1985). The heliocentic radial velocity was $\dot{r}=$
$26.6 \mathrm{~km} \mathrm{~s}^{-1}$. The closest approach of the comet to the Earth occured on April 11, 1986 (West 1988).

The observation geometry as projected on the celestial plane is shown in Fig. 1. Superimposed upon a contour plot of the coma of $\mathrm{P} /$ Halley this figure contains the projected directions from the cometary nucleus towards north $(\mathrm{N})$, east $(\mathrm{E})$, and the sun $(\odot)$. The proper motion $(\mathrm{PM})$ of the comet was about $0.19 \operatorname{arcsec~s} \mathrm{s}^{-1}$. The position angle (counted N-E-S-W) of the sun was equal to $135.7^{\circ}$, and that of the proper motion vector was $273.0^{\circ}$. Further details of this figure will be explained in Sect. 2.3.

The phase angle of $\mathrm{P} / \mathrm{Halley}$, i.e. the angle sun-cometearth, had a value of $\alpha=34.3^{\circ}$. This caused an apparent shortening of the comet tail in the projection on the celestial plane. 
Table 1. Processed plates obtained on April 10, 1986, and related data

\begin{tabular}{|c|c|c|c|c|c|c|c|c|c|}
\hline \multirow[t]{2}{*}{ Plate } & \multirow[t]{2}{*}{ Object } & \multicolumn{2}{|c|}{ Exposure } & \multirow[b]{2}{*}{ Factor $^{b}$} & \multicolumn{3}{|c|}{ Instrumentation } & \multirow[t]{2}{*}{ Airmass $^{\mathrm{d}}$} & \multirow[t]{2}{*}{ Data set } \\
\hline & & $\begin{array}{l}\text { Begin } \\
(\mathrm{UT})\end{array}$ & $\begin{array}{l}\text { Time } \\
\text { (s) }\end{array}$ & & Filter & $\mathrm{SP}^{\mathrm{c}}$ & $\mathrm{GP}^{\mathrm{c}}$ & & \\
\hline $\begin{array}{c}\text { F161 } \\
\ldots a\end{array}$ & $\begin{array}{c}\mathrm{P} / \text { Halley } \\
"\end{array}$ & $\begin{array}{l}014630 \\
015100\end{array}$ & $\begin{array}{l}180 \\
60\end{array}$ & & $\begin{array}{l}\text { IF } 369 \\
\text { BG } 24\end{array}$ & $\mathrm{x}$ & & 1.994 & \\
\hline $\begin{array}{l}\text { F162 } \\
\text { F163 } \\
\text { F164 }\end{array}$ & $\begin{array}{l}\text { P/Halley } \\
\text { P/Halley } \\
\text { P/Halley }\end{array}$ & $\begin{array}{l}015530 \\
022700 \\
023800\end{array}$ & $\begin{array}{l}1800 \\
600 \\
180\end{array}$ & $\begin{array}{l}.37(6) \\
2.8(4)\end{array}$ & $\begin{array}{l}\text { BG } 24 \\
\text { BG } 24 \\
\text { BG } 24\end{array}$ & $\begin{array}{l}\mathrm{x} \\
\mathrm{x} \\
\mathrm{x}\end{array}$ & $\begin{array}{l}x \\
x \\
x\end{array}$ & $\begin{array}{l}1.793 \\
1.640 \\
1.594\end{array}$ & $\mathrm{~A}$ \\
\hline $\begin{array}{l}\text { F166 } \\
"\end{array}$ & P/Halley & $\begin{array}{l}031630 \\
031800\end{array}$ & $\begin{array}{l}30 \\
360\end{array}$ & & $\begin{array}{l}\text { BG } 24 \\
\text { IF } 369\end{array}$ & $\mathrm{x}$ & & 1.390 & \\
\hline $\begin{array}{l}\text { F167 } \\
\text { F168 } \\
\text { F169 }\end{array}$ & $\begin{array}{l}\text { P/Halley } \\
\text { P/Halley } \\
\text { P/Halley }\end{array}$ & $\begin{array}{l}032600 \\
040100 \\
041200\end{array}$ & $\begin{array}{l}1800 \\
600 \\
180\end{array}$ & $2.9(6)$ & $\begin{array}{l}\text { BG } 24 \\
\text { BG } 24 \\
\text { BG } 24\end{array}$ & $\begin{array}{l}\mathrm{x} \\
\mathrm{x} \\
\mathrm{x}\end{array}$ & $\begin{array}{l}x \\
x \\
x\end{array}$ & $\begin{array}{l}1.317 \\
1.242 \\
1.223\end{array}$ & B \\
\hline F170 & P/Halley & 044900 & 360 & & IF 369 & & & 1.144 & \\
\hline $\begin{array}{l}\mathrm{F} 171 \\
\quad "\end{array}$ & $\begin{array}{c}\mathrm{P} / \text { Halley } \\
\text {, }\end{array}$ & $\begin{array}{l}045600 \\
050245\end{array}$ & $\begin{array}{l}360 \\
30\end{array}$ & & $\begin{array}{l}\text { IF } 369 \\
\text { BG } 24\end{array}$ & $\mathrm{x}$ & & 1.132 & \\
\hline $\begin{array}{l}\text { F172 } \\
\text { F173 } \\
\text { F174 }\end{array}$ & $\begin{array}{l}\mathrm{P} / \text { Halley } \\
\mathrm{P} / \text { Halley } \\
\mathrm{P} / \text { Halley }\end{array}$ & $\begin{array}{l}050500 \\
053630 \\
054830\end{array}$ & $\begin{array}{l}1806 \\
600 \\
180\end{array}$ & $\begin{array}{l}.35(5) \\
2.6(5)\end{array}$ & $\begin{array}{l}\text { BG } 24 \\
\text { BG } 24 \\
\text { BG } 24\end{array}$ & $\begin{array}{l}\mathrm{x} \\
\mathrm{x} \\
\mathrm{x}\end{array}$ & $\begin{array}{l}\mathrm{x} \\
\mathrm{x} \\
\mathrm{x}\end{array}$ & $\begin{array}{l}1.104 \\
1.081 \\
1.074\end{array}$ & $\mathrm{C}$ \\
\hline $\begin{array}{c}\text { F175 } \\
\text { " }\end{array}$ & $\begin{array}{c}\mathrm{P} / \text { Halley } \\
,\end{array}$ & $\begin{array}{l}060130 \\
060900\end{array}$ & $\begin{array}{l}360 \\
60\end{array}$ & & $\begin{array}{l}\text { IF } 369 \\
\text { BG } 24\end{array}$ & $\mathrm{x}$ & & 1.064 & \\
\hline $\begin{array}{l}\text { F176 } \\
\text { F177 } \\
\end{array}$ & $\begin{array}{c}\text { NGC } 6302 \\
\text { NGC } 6302 \\
\quad,\end{array}$ & $\begin{array}{l}071700 \\
073300 \\
073600\end{array}$ & $\begin{array}{l}600 \\
60 \\
360\end{array}$ & & $\begin{array}{l}\text { BG 24 } \\
\text { BG } 24 \\
\text { IF } 369\end{array}$ & $\begin{array}{l}\mathrm{x} \\
\mathrm{x}\end{array}$ & $\mathrm{x}$ & 1.052 & \\
\hline
\end{tabular}

a Quotation marks indicate a double exposure.

b Computed factor with rmse to adjust the exposure time to $600 \mathrm{~s}$.

${ }^{c}$ A cross indicates whether the slit plate (SP) resp. the grating prism (GP) was used during exposure.

d Airmass for mean exposure time.

\subsection{Instrumentation}

The observations were performed at the ESO $1 \mathrm{~m}$ telescope using the focal reducer of the Observatory Hoher List (Geyer et al. 1979) and instrumental components provided by the Max-Planck-Institut für Aeronomie. The instrumentation has been described by Jockers et al. (1987).

After having passed the Cassegrain focus of the telescope the beam is recollimated. Filters (imaging mode) or grating prisms (spectrographic mode) are placed in the parallel beam. A camera lens with $50 \mathrm{~mm}$ aperture and $140 \mathrm{~mm}$ focal length creates a reduced image of the Cassegrain focus at the detector. In imaging mode at the ESO $1 \mathrm{~m}$ telescope, the plate scale was 67.6 arcsec $\mathrm{mm}^{-1}$ and the field size about $22 \times$ $22 \operatorname{arcmin}^{2}$. In this mode an interference filter centered at $3690 \AA$ with half width (FWHM) of $\approx 100 \AA$ was used which shows the cometary coma predominantly in the light of $\mathrm{CO}_{2}^{+}$.

In spectrographic mode a slit plate consisting of 69 slits arranged in five columns was employed in the Cassegrain focal plane as diaphragm to simultaneously obtain spectra from 69 different parts of the coma of $\mathrm{P} / \mathrm{Halley}$. The grating prism had 600 grooves per $\mathrm{mm}$ and was used in first order. An overlap of individual spectra was avoided by an interlaced arrangement of the slits combined with a slight tilt between the slit direction and the normal with respect to the grating dispersion. At the first order straight-through wavelength $(3930 \AA)$ the reciprocal linear dispersion was about $100 \AA \mathrm{mm}^{-1}$. The exact value of dispersion depended on the positions of the slits relative to the grating prism (Loewen 1970). The spectral resolution was about $4 \AA$. The focal reducer lens optics and a BG 24 filter restricted the effective wavelength range to the interval between approximately 3500 and $4500 \AA$.

The detector consisted of a two stage proximity image intensifier with bi-alkali cathode (Cromwell et al. 1985) and plates pressed against its output window of $25 \mathrm{~mm}$ diameter. The advantage of the image intensifier in comparison with a CCD camera was its better sensitivity in the near-UV and blue spectral ranges where principal 
cometary emissions are located. The main disadvantage of the intensifier was its large-scale signal-induced background (Cromwell \& Smith 1973). The intensifier created halos surrounding the brightest areas and a fixed pattern of small spots. All images were recorded on hypersensitized IIIa-F plates of $45 \times 45 \mathrm{~mm}^{2}$ size. Inhomogeneities in the hypersensitation may be present but probably are of minor influence. The weather conditions allowed measurements of high photometric quality.

\subsection{Observational method and plates}

The aim of the observations was to obtain spectra of the comet from different coma areas. In order to know the locations of the slits with respect to the changing plasma tail, first a filter image of $\mathrm{P} /$ Halley was obtained in the light of $\mathrm{CO}_{2}^{+}$using an interference filter centered at $3690 \AA$ with $100 \AA$ full width at half maximum (FWHM). This filter shows the plasma tail best, because the contribution of dust continuum and neutral coma is reduced. Then the interference filter was changed into a BG 24 filter and the slit plate was inserted to record the slit positions in double exposure technique on the same plate. After that the focal reducer was used with the grating prism and three multislit spectra were taken with different exposure times in order to enlarge the dynamic range of measurable cometary intensities. This cycle was repeated three times, i.e. altogether three data sets $\mathrm{A}, \mathrm{B}$, and $\mathrm{C}$, were recorded, each consisting of four plates. The plates are listed in Table 1. Pictures of plates F171 and F172 were presented by Jockers et al. (1987). During the cometary exposures the telescope tracking was set to follow the motion of the comet.

In addition a single-exposure direct image of $\mathrm{P} / \mathrm{Halley}$ without slit marks was recorded on plate F170. In Fig. 1 a contour plot of this image is shown in logarithmic intensity scale without any background subtracted. The head of $\mathrm{P} /$ Halley and the $\mathrm{CO}_{2}^{+}$ion tail, pointing away from the sun, are visible. In this figure the slit positions for the three data sets, deduced from plates F161, F166, and F171, are represented by short vertical lines. For each data set they are arranged in five columns. The slits are aligned parallel to the north-south direction and their projected size at the comet is about $850 \times 9100 \mathrm{~km}^{2}$. The covered field size is about $410^{5} \times 410^{5} \mathrm{~km}^{2}$. The location of the slit pattern is slightly shifted in the data sets A, B, and C. The reason for this is either the bending within the instrument or tracking errors of the telescope. In the data analysis we have assumed that the image of the slits with respect to the comet taken at the beginning of the data set is representative for the location during acquisition of the spectra. For data set $\mathrm{C}$ this is proven by the additional exposure F175, on which the position of the slits is identical to F171. Furthermore Fig. 1 shows trailed background stars and the circular output window of the image inten- sifier. In the upper part at the left side a large hot spot of the intensifier is visible which appears on all plates.

For absolute calibration a spectrum (F176) of the emission line nebula NGC 6302 (Aller et al. 1981) was recorded. The measured relative line intensities were compared to published absolute intensities, and the derived response function was used to calibrate the cometary spectra. Image F177 shows the location of the slit with respect to the nebula and indicates that the brightest area was missed by about 15 arcsec. CCD spectra of NGC 6302 were taken in July 1982 with the ESO $1.5 \mathrm{~m}$ telescope at the brightest part of the nebula and at the slit location indicated by plate F177. These data indicate that spectrum F176 represents well the brightest part of the nebula. In view of the uncertainty in telescope tracking and the small angular extent of the nebula we have assumed that the brightest part of the nebula was recorded in the plate spectrum. If this was not the case, our cometary column densities must be considered as upper limits. As we will see in Sect. 5.1 our values are indeed higher than the values found in the literature, but by not more than a factor of 2 .

\section{Data reduction}

The plates were digitized in May 1992, at ESOHeadquarters in Garching, Germany, using a PDS 1010A microdensitometer (Eccles et al. 1983) with an aperture of $20 \times 20 \mu \mathrm{m}^{2}$. The direct filter images were scanned in $20 \mu \mathrm{m}$ steps in each direction. In the scans of the multislit spectra the steps in the direction perpendicular to the slits was reduced to $10 \mu \mathrm{m}$, i.e. the spectra were oversampled in dispersion direction.

\subsection{Processing the digitized images}

Characteristic curve. The characteristic curve of the plates (see Eccles et al. 1983) was determined by a sensitometer calibration. The calibration plate was exposed $600 \mathrm{~s}$ using the ESO tube spot sensitometer with a BG 18/OG 515 filter combination approximating the colour of the phosphor output window of the image intensifier.

Alignment of images. One direct image with the slit pattern superimposed on the comet images was rotated to align the slits to the vertical image axis (see Fig. 1). Then all images were adjusted relative to each other by making use of the fixed pattern of the small intensifier spots, existing on all plates. The accuracy of this reduction step in terms of spectral resolution was about $1 \AA$.

Adjustment of exposure times. The relative intensities of the 3 and 30 min. exposures of each data set were adjusted to the intensities of the $10 \mathrm{~min}$. exposure. The relevant exposure times (Table 1) gave adjustment factors of $3 . \overline{3}$ and $0.3 \overline{3}$, respectively. For verification, the intensity values of corresponding pixels in the spectra images were directly compared. Only pixels belonging to a spectrum 


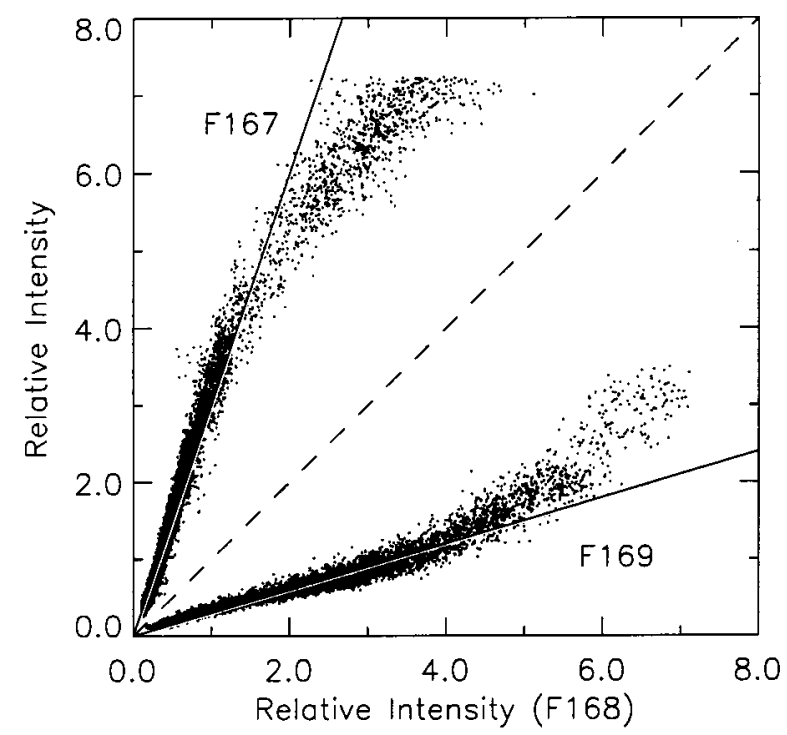

Fig. 2. Comparison of corresponding pixel values in processed images F167, F168, and F169 with 30, 10, and 3 min exposure

and the reliable intensity interval of the characteristic curve were selected, pixels of the plate background and of detector blemishes were rejected. For data set B the result is shown in Fig. 2. About $10^{5}$ pixel values of the images F167 and F169 are plotted against the value of the corresponding pixels in image F168. In Fig. 2 the slopes of the straight lines are the exposure time ratios. The deviations from linearity are probably caused by the characteristic curve underestimating the values of the higher intensities. Furthermore, the different airmasses (Table 1) in combination with the coefficient of extinction may result in some wavelength dependence of the factors. Therefore, instead of the exposure time ratios empirical factors (Table 1 ) deduced from the pixel comparison itself were applied to the images. Figure 2 indicates that, after adjusting the exposure times, image F167 was the more important source for low intensities while F169 was more important for high intensities, i.e. the dynamic intensity range of image F168 was effectively enlarged.

Average of images. The averaged multislit spectra image $A$ of a data set was calculated from the three corresponding spectra images $I_{1}, I_{2}$, and $I_{3}$, using the equation

$$
A=\sum\left(W_{\mathrm{i}} \cdot I_{\mathrm{i}}\right) / \sum W_{\mathrm{i}} \quad \text { with } \quad W_{\mathrm{i}}=M_{\mathrm{i}} / E_{\mathrm{i}}^{2} .
$$

The weights $W_{\mathrm{i}}$ were formed out of mask images $M_{\mathrm{i}}$ and error images $E_{\mathrm{i}}$ which were created for every spectrum image $I_{\mathrm{i}}$. The pixel values of the mask images were set to 1 and 0 , respectively, depending on wether the related pixels in the spectra images contained useful spectral information or not. This way, pixel values contaminated by background stars, the fixed pattern of the intensifier spots, scratches on some plates, or limitations caused by the characteristic curve, were marked with 0 . The pixel values in the error images were estimated errors of the corresponding pixel values in the spectra images. These error images were created just after applying the characteristic curve to the digitized images, and were processed in the same way.

\subsection{Extraction and calibration of the spectra}

Extraction of spectra. The skew between the slit direction and the dispersion in the averaged multislit spectra images was removed by shearing these images parallel to the slits by an angle of $2.35^{\circ}$. One-dimensional spectra were then extracted by averaging up to 15 pixel values in slit direction. In Fig. 3a an extracted cometary head spectrum is shown. The related slit position is marked in Fig. 1 with an arrow.

Subtraction of plate background. To correct for the large-scale intensifier background on the plates the nearby emission-free area surrounding each spectrum was used to approximate the individual background for that spectrum. In Fig. 3a this approximation is shown as a thin line below the spectrum.

Wavelength calibration. The wavelength calibration was done by identifying spectral emission features of known wavelengths in the cometary spectra and comparing them with maximum intensity wavelengths published by Swings \& Haser (1956). The reciprocal linear dispersion of the five spectra columns of data set $\mathrm{C}$ for example resulted in 95.2, 94.1, 93.5, 92.1, and $89.8 \AA \mathrm{mm}^{-1}$, respectively. The wavelength co-ordinate was rectified with a polynomial of first degree. The NGC 6302 spectrum was extracted the same way as the Halley spectra, but for the wavelength calibration the publications of Aller \& Czyzak (1978) and Aller et al. (1981) were used.

Intensity calibration. The extracted spectra were corrected for extinction using the computed airmasses of the the plates with $600 \mathrm{~s}$ exposure (Table 1) and the nominal ESO coefficient of extinction published by Danks (1983). The intensities of the spectra were normalized to $1 \mathrm{~s}$. To determine the response function of the spectra, the relative line intensities of eight NGC 6302 emission features were integrated and compared to known absolute line intensities (Fig. 3b). For this purpose, relative intensities from Oliver \& Aller (1969), Aller \& Czyzak (1978), and Aller et al. (1981), were calibrated using absolute intensities for $\mathrm{H} \delta, \mathrm{H} \gamma$, and [OIII], which were deduced from Danziger et al. (1973). The response function was approximated by a Gaussian curve. The calibrated example spectrum is shown in Fig. 3c.

\subsection{Derivation of absolute intensities}

Dust continuum. In the reduced spectra a contribution of the solar spectrum is visible which is backscattered from the cometary dust grains. In order to model this dust continuum and to create a solar spectrum $I_{\odot}(\lambda)$ with the 


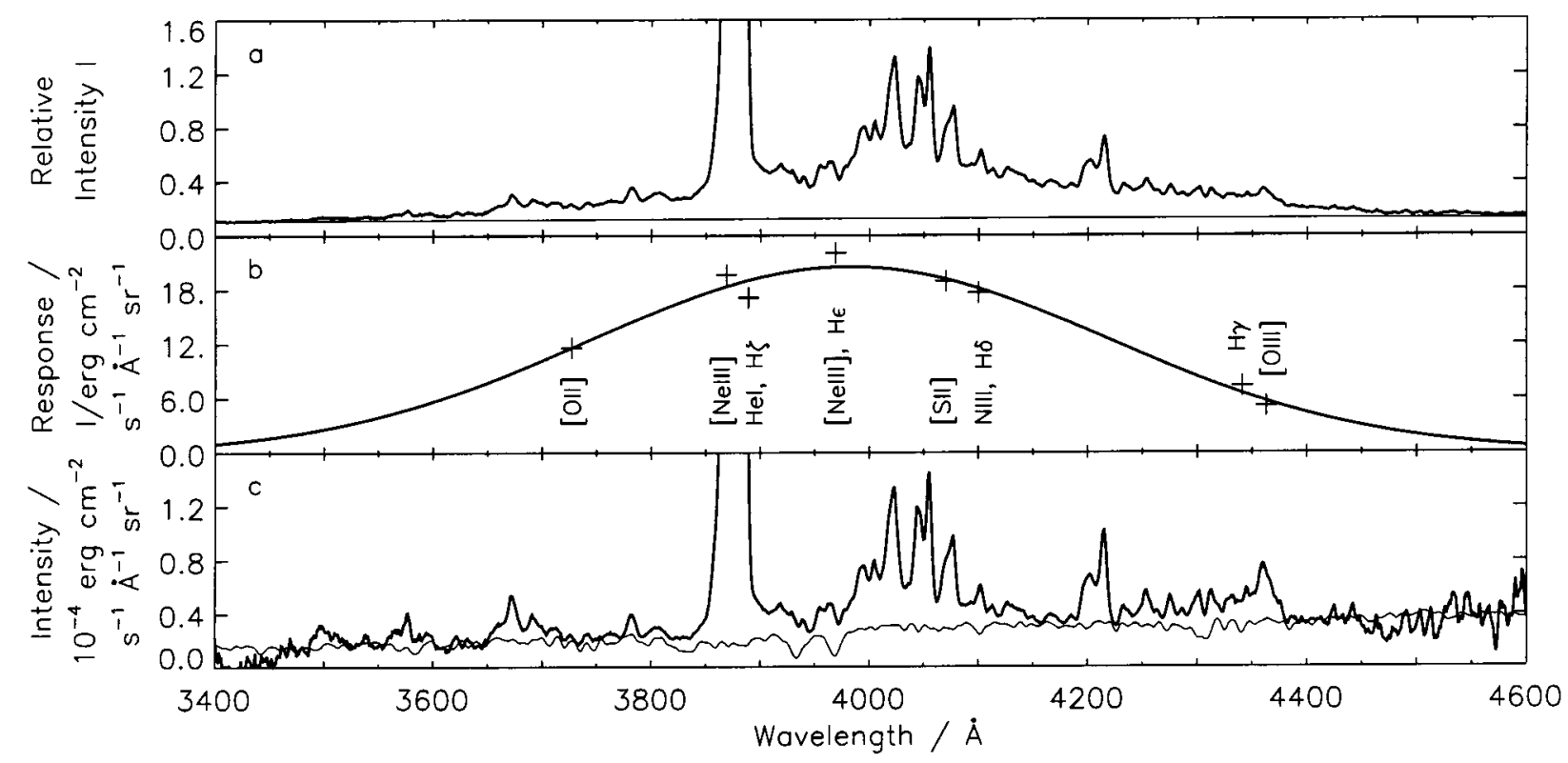

Fig. 3. Steps in the data reduction of a cometary head spectrum (marked by an arrow in Fig. 1): Extraction a), calibration b), and approximation of the dust continuum c)

resolution of the instrument, the FWHM of the cometary spectra $(5.2 \AA)$ was determined from the well resolved emission lines of the NGC 6302 spectrum. Then the solar spectrum published by Kurucz et al. (1984) was convolved with the corresponding Gaussian curve to adjust the spectral resolution. The reddening of the dust continuum of $\mathrm{P} /$ Halley with respect to the solar spectrum was considered by applying the following equation of Werner et al. (1989), which is in accordance with the color values presented by Meech \& Jewitt (1987) and Thomas \& Keller (1989):

$I_{\text {Continuum }}(\lambda) \propto \sqrt{\lambda} \cdot I_{\odot}(\lambda)$

The resulting spectrum was adjusted to the cometary spectra in a way, that after its subtraction a maximum continuum contribution was removed without leading to negative intensities in the wavelength range between 3750 and $4350 \AA$. The strength of each approximated dust continuum was then measured in the continuum window at $3650 \AA$. An adjusted continuum is shown in Fig. 3c. Even around $3600 \AA$ the approximation still fits the dust continuum very well.

Molecular bands. To identify the emission features in the cometary spectra they were compared with theoretical and laboratory wavelengths and observed spectra (Gerö 1941; Mrozowski 1947a,b; Swings \& Haser 1956; Gausset et al. 1965; Brocklehurst et al. 1972; McCallum \& Nicholls 1972; Zucconi \& Festou 1985; Arpigny et al. 1986a,b; Magnani \& A'Hearn 1986; Jockers et al. 1987; Wyckoff \& Theobald 1989; Valk et al. 1992; Kim \& A'Hearn 1993, pers. communication; Lutz et al. 1993). The extracted spectra show a large number of cometary emissions of different sources, but for the present paper the stronger and sufficiently resolved emissions rather than tentative identifications were considered. The selected emissions with their peak wavelengths and integration intervals are listed in Table 2 and marked in Fig. 4. If possible, we did not rely on the continuum subtraction described in the previous paragraph, but interpolated a so-called pseudocontinuum between long and short wavelength side of the molecular emission. In the case of $\mathrm{CO}_{2}^{+}$the band intensity was integrated with and without considering a pseudocontinuum. The emission of $\mathrm{C}_{3}$ around $4050 \AA$ is mainly contaminated by the $\mathrm{CO}^{+}(3-0)$ emission, and therefore its integration range was reduced to exclude this contamination. The integrated $\mathrm{C}_{3}$ intensities were multiplied with 2.12 to adjust for the whole $\mathrm{C}_{3}$ band. This factor was deduced from the strongest head spectrum where the $\mathrm{CO}^{+}(3-0)$ contamination could be neglected. No emissions of the night sky were found in the spectra.

\subsection{Molecular column densities}

The integrated molecular band intensities $I$ were transformed to column densities $N$ using the fluorescence emission rates $g$ listed in Table 2 and the equation

$\log _{10}(N)=\log _{10}\left(\frac{4 \pi}{g(r)}\right)+\log _{10}(I)$

(see Lutz et al. 1993). The used emission rate for $\mathrm{CO}_{2}^{+}$is preliminary and may be revised in the future. 
Table 2. Investigated emissions in spectra of $\mathrm{P} / \mathrm{Halley}$, and related data

\begin{tabular}{lllll}
\hline Identification & $\begin{array}{l}\text { Wavelengths }^{\mathrm{a}} \\
(\AA)\end{array}$ & $\begin{array}{l}\text { Integration } \\
(\AA)\end{array}$ & $\begin{array}{l}g^{\mathrm{b}} \\
\left(10^{-12} \mathrm{erg} \mathrm{s}^{-1}\right)\end{array}$ & $\begin{array}{l}\log _{10}\left(\frac{4 \pi}{g(r)}\right)^{\mathrm{c}} \\
\left(\mathrm{erg}^{-1} \mathrm{~s}\right)\end{array}$ \\
\hline $\mathrm{CN}(0-0)$ & 3882.02 & $3843-3894$ & $.458^{\mathrm{f}}$ & 13.68 \\
$\mathrm{C}_{3}$ & $3936.5^{\mathrm{e}}, 3963.5,3992.5,4019.4$, & $4037-4115^{\mathrm{I}}$ & $1 .^{\mathrm{g}}$ & 13.34 \\
$\mathrm{CN}(0-1)$ & $4043.6,4051.6,4074.4,4099.5$ & & \\
$\mathrm{CH}(0-0) \mathrm{AX}$ & 4214.7 & $4190-4223^{\mathrm{P}}$ & $.023^{\mathrm{h}}$ & 14.98 \\
\hline $\mathrm{CO}_{2}^{+}(0-1),(1-2)$ & $4301^{\mathrm{d}}, 4313.2$ & $4294-4320^{\mathrm{P}}$ & $.1^{\mathrm{i}}$ & 14.34 \\
$\mathrm{CO}_{2}^{+}(0-1),(1-2)^{\mathrm{P}}$ & 3674,3695 & $3660-3700$ & $.012^{\mathrm{j}}$ & 15.26 \\
$\mathrm{CO}^{+}(4-0)$ & $3781.3,3802.5$ & $3660-3700^{\mathrm{P}}$ & $"$ & $"$ \\
$\mathrm{CO}^{+}(3-0)$ & $4001.5,4024$ & $3771-3810^{\mathrm{P}}$ & $.011^{\mathrm{k}}$ & 15.30 \\
$\mathrm{CO}^{+}(2-0)$ & $4250.9,4273.8$ & $3997-4033^{\mathrm{P}}$ & $.021^{\mathrm{k}}$ & 15.02 \\
\hline
\end{tabular}

P Pseudocontinua were subtracted before integration.

I Integration results were transformed to the wavelength range $3925-4115 \AA$.

a All but one after Swings \& Haser (1956).

b Valid for $r=1 \mathrm{AU}$ and $\dot{r}=0 \mathrm{~km} \mathrm{~s}^{-1}$, but in the cases of $\mathrm{CN}$ and $\mathrm{CO}^{+}$valid for $\dot{r} \approx 27 \mathrm{~km} \mathrm{~s}^{-1}$

c The fluorescence emission rates $g$ were scaled to $r=1.32 \mathrm{AU}$ using a $r^{-2}$ law.

d Mean wavelength of related rotational subbands.

\section{Spectra}

Representative spectra of comet $\mathrm{P} /$ Halley are shown in Figs. 4a-f. These spectra were created by averaging spectra from selected coma areas in order to enlarge their signal-to-noise ratio. The slit positions of the selected spectra are marked in Figs. 4g-l by filled circles. The averaged spectra were normalized to the band intensity of their CN $(0-1)$ emission (see scaling factors on the intensity axis of Figs. 4a-f).

The averaged tail spectrum in Fig. 4d reveals the dominant plasma tail emissions at a mean projected distance from the nucleus of about $\bar{\rho}=10^{5.1} \mathrm{~km}$. The $(4-0)$, $(3-0)$, and $(2-0)$, double bands of the $\mathrm{CO}^{+}$Comet Tail System and the $(0-1)$ and $(1-2)$ bands of the $\mathrm{CO}_{2}^{+}$Fox Duffendack Barker System were identified. Additionally, there is a large number of emissions heavily blended or too weak to be measured. The peak around $3575 \AA$ is a blend of emissions from $\mathrm{CN}, \mathrm{CO}^{+}, \mathrm{CO}_{2}^{+}, \mathrm{N}_{2}^{+}$, and $\mathrm{OH}^{+}$. The latter emission should be the dominating contribution in the present case (see Wyckoff \& Theobald 1989; Arpigny et al. 1986b). The $(0-0)$ band of the $\mathrm{N}_{2}^{+}$First Negative System at $3914 \AA$ is the strongest emission in this system (Lutz et al. 1993). But in the present spectra it is fairly weak and contaminated by other emissions (see Wyckoff $\&$ Theobald 1989). The peak at $4235 \AA$ represents the $\mathrm{CH}^{+}(0-0) \mathrm{AX}$ band which is the strongest $\mathrm{CH}^{+}$emission in the observed spectral range (Lutz et al. 1993), but rather weak in most spectra. The emission around 4130

\author{
e Valk et al. (1992). \\ f Tatum (1984). \\ g A'Hearn (1982). \\ h Zucconi \& Festou (1985). \\ ${ }^{i}$ Lutz et al. (1993). \\ j deduced from data provided by \\ Kim \& A'Hearn (1993, pers. communication). \\ k Magnani \& A'Hearn (1986).
}

$\AA$ could not be identified with certainty (see Jockers et al. 1987). Interestingly, the ion emissions, especially the $\mathrm{CO}^{+}$ $(2-0)$ band, are not only limited to the plasma tail but are also visible in the head spectrum (Fig. 4b) and even in the spectrum of the sunward coma (Fig. 4a). This suggests the presence of a large $\mathrm{CO}^{+}$halo around the comet (see also Jockers et al. 1987; Jockers \& Bonev 1997).

In contrast to the tail spectrum, the head spectrum at $\bar{\rho}=10^{4.2} \mathrm{~km}$ is dominated by neutral gas emissions. Most prominent is the Violet System of $\mathrm{CN}$ with its $(0-0)$ and $(0-1)$ bands which are visible in all spectra. The rich $\mathrm{C}_{3}$ emission around $4050 \AA$ has eight certainly identifiable peaks which are partly blended with weaker emissions of $\mathrm{N}_{2}^{+}, \mathrm{CH}^{+}, \mathrm{CO}^{+}$, and $\mathrm{CO}_{2}^{+}$(see Wyckoff \& Theobald 1989). In our spectra, there is some evidence for $\mathrm{C}_{3}$ also contributing to the emissions at wavelengths shorter than CN $(0-0)$ (see Gausset et al. 1965; Valk et al. 1989). Finally, the $\mathrm{CH}$ radical is represented by its $(0-0) \mathrm{AX}$ band. The $\Delta \nu=2$ band of the $\mathrm{C}_{2}$ Swan band system around $4360 \AA$ is much weaker than the other bands of that system which were analysed with regard to spatial distribution by several other authors (see e.g. Kidger et al. 1987; Combi \& Fink 1993).

\section{Profiles}

\subsection{Intensity and column density profiles}

Intensity profiles. The observed intensity profiles of the dust continuum and the neutral radicals are shown in 

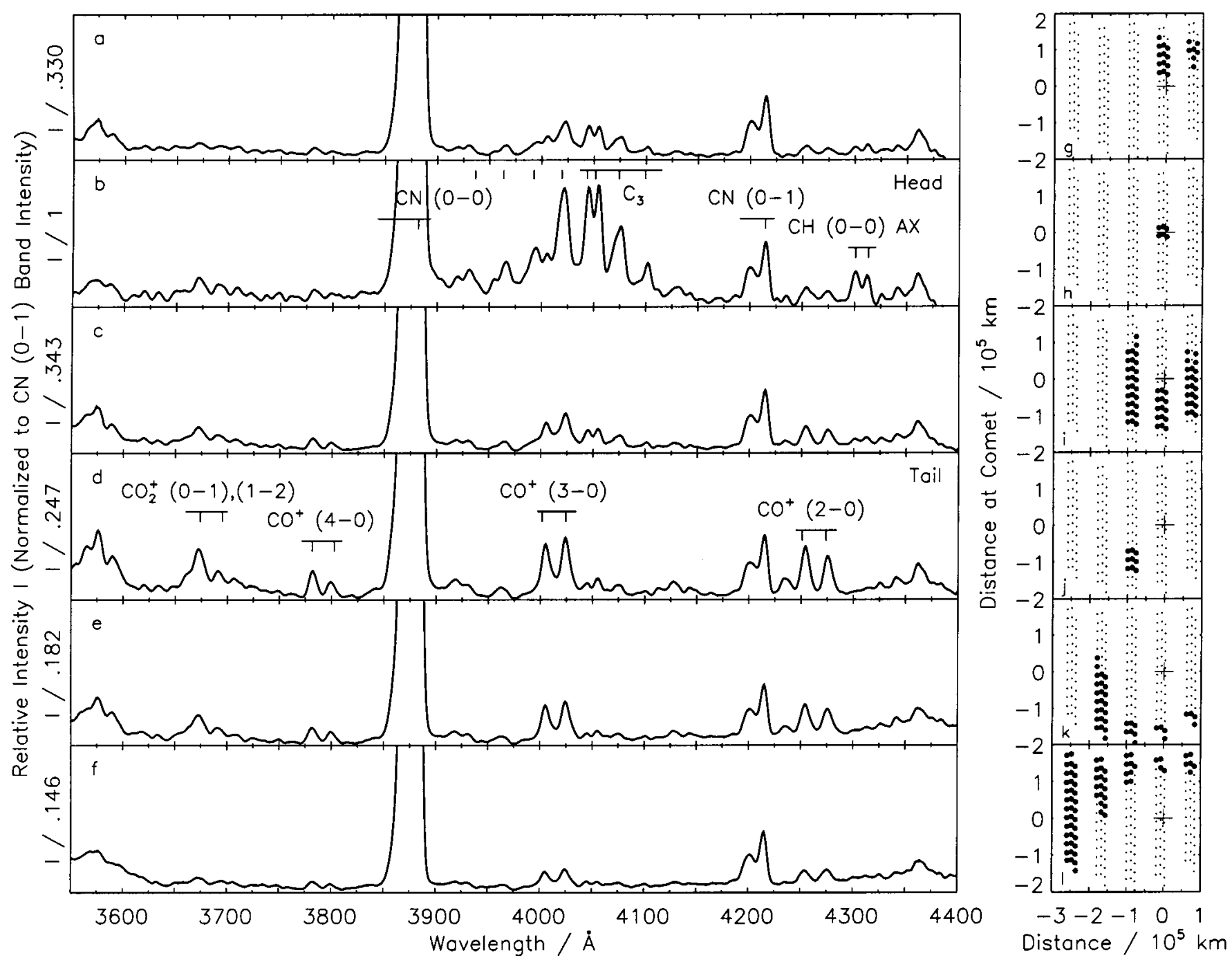

Fig. 4. Synopsis of spectra a-f) averaged over different areas of the cometary coma (compare panels g-l) with Fig. 1)

Fig. 5 in double logarithmic presentation. Similar profiles for the molecular ions are presented in Fig. 6. The continuum and molecular band intensities $I$ are plotted as a function of the projected distance from the nucleus $\rho$. As there is no temporal variation apparent in the three data sets (see Figs. 8 and 9) the intensities were plotted without any distinction between the three data sets. To provide some spatial resolution, the data were divided into four classes of profiles: sunward, coma, tailward, and perpendicular to tail. Obviously incorrect intensity values were not plotted.

The figure panels 5a-k and 6a-o show in each row the same intensity values of one of the considered species in the form of black and grey dots. The grey, highlighted dots represent the selected values, i.e. the values belonging to the slit positions of the sunward, coma, and tailward areas in Fig. 5a-k and the sunward, perpendicular to tail, and tailward areas in Fig. 6a-o. The thin line is a fit to the highlighted, i.e. selected values. The selected slit positions are indicated for every profile in the panels 5l-p and $6 \mathrm{p}-\mathrm{t}$, respectively.

The intensity profiles of the dust continuum at $3650 \AA$ (Fig. 5a-c) are expected to follow a $\rho^{-1}$ law (radial expansion of a particle cloud with constant speed). The deduced gradient of the overall coma profile $(-1.1)$ is consistent with the expected value $(-1)$ and with other observations for P/Halley (e.g. Levasseur-Regourd et al. 1986; Gammelgaard \& Thomson 1988). The gradient of the tailward profile $(-.86)$ is consistent with the $\rho^{-1}$ law, too, whereas the sunward gradient $(-1.6)$ shows a significant deviation. Jewitt \& Meech (1987) also found steeper gradients than -1 for $\mathrm{P} /$ Halley. As an explanation Ellis \& Neff (1992) considered temporal and spatial variations in the dust production rate of $\mathrm{P} / \mathrm{Halley}$ itself.

The strength of the continuum is given in Table 3 in units of mean solar disc intensities (Allen 1973). 

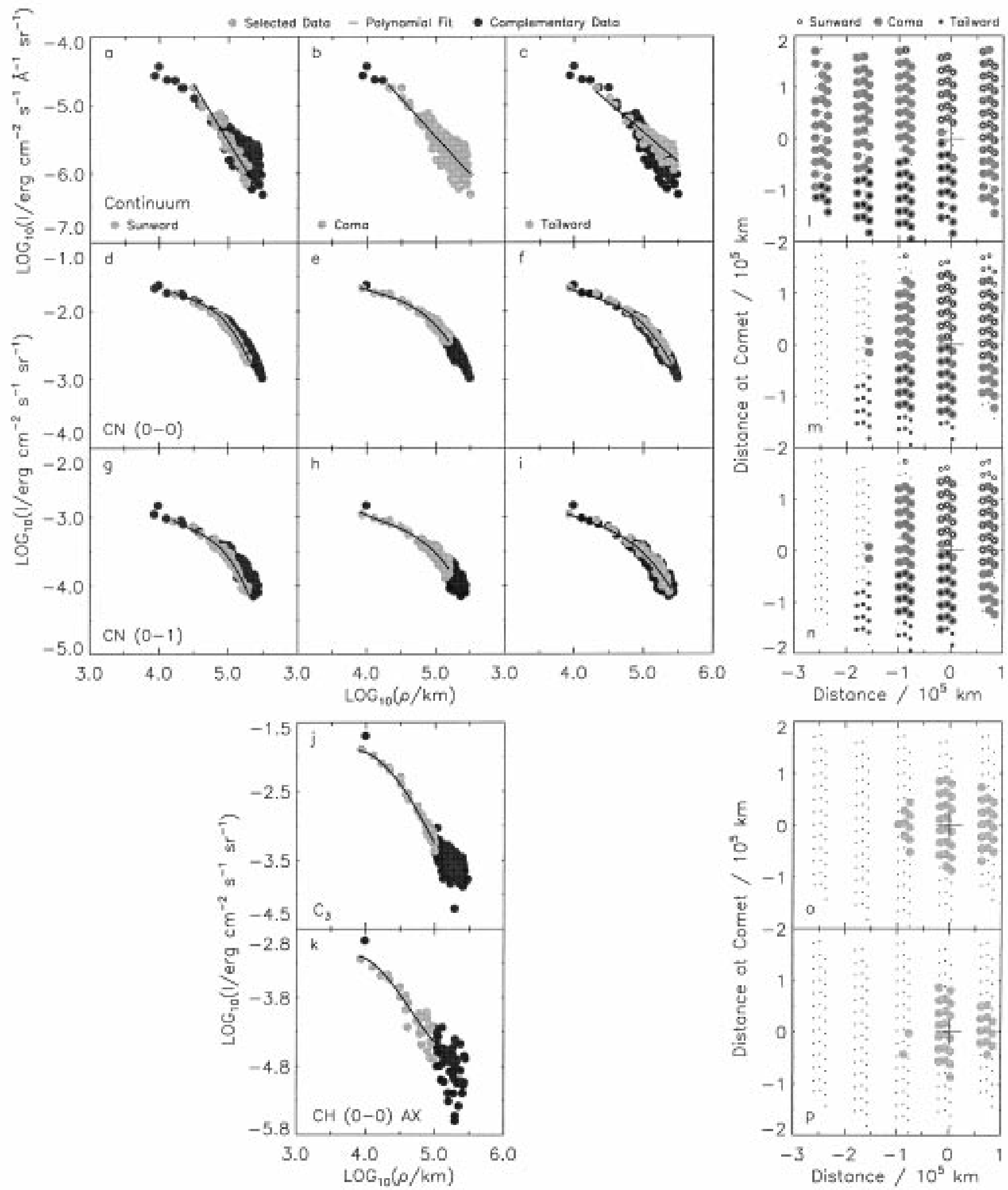

Fig. 5. Radial intensity profiles of neutral coma constituents (a-k) for different areas of the coma (compare panels (l-p) with Fig. 1) 

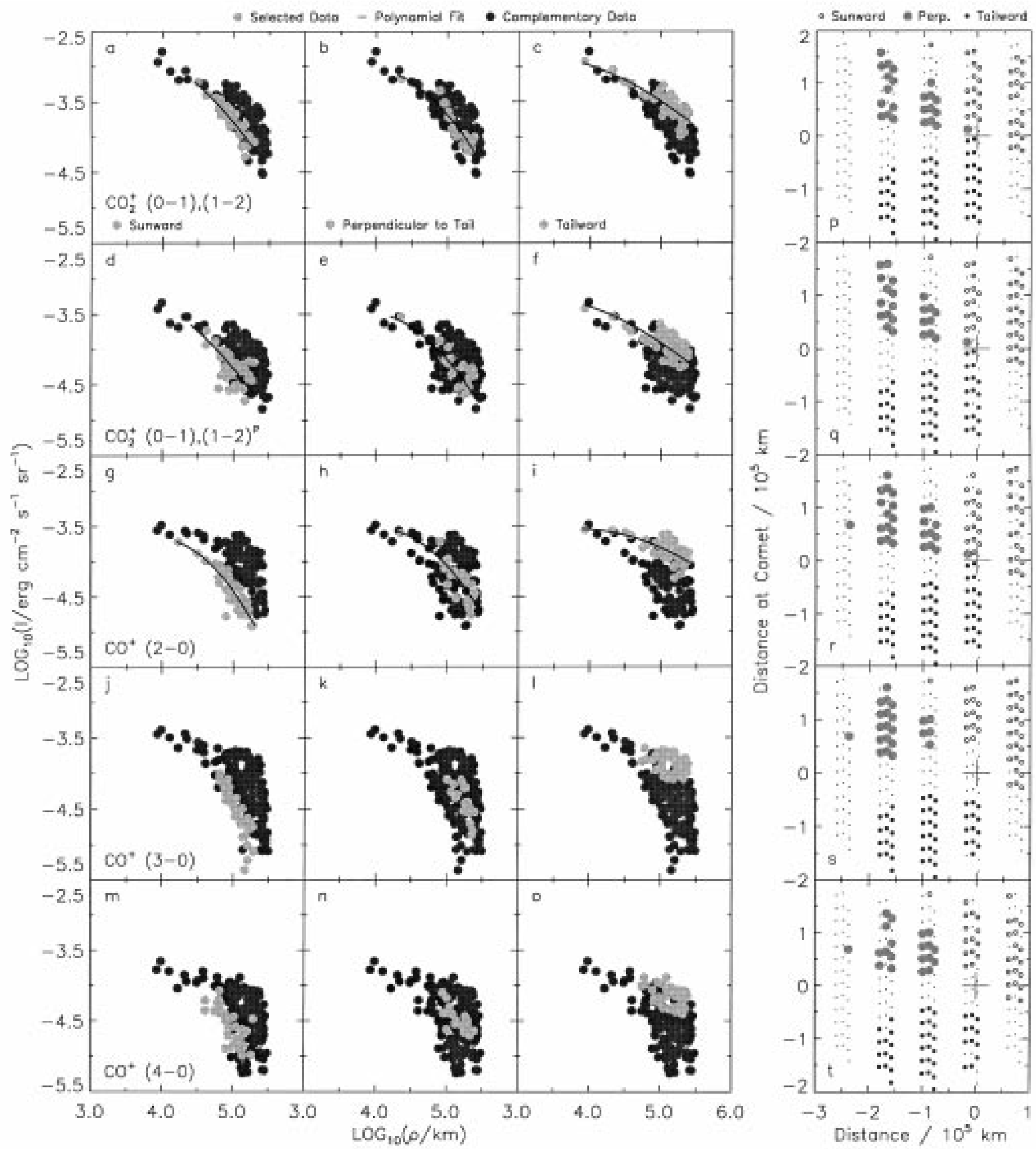

Fig. 6. Radial intensity profiles of ionic coma constituents (a-o) for different areas of the coma (compare panels (p-t) with Fig. 1) 


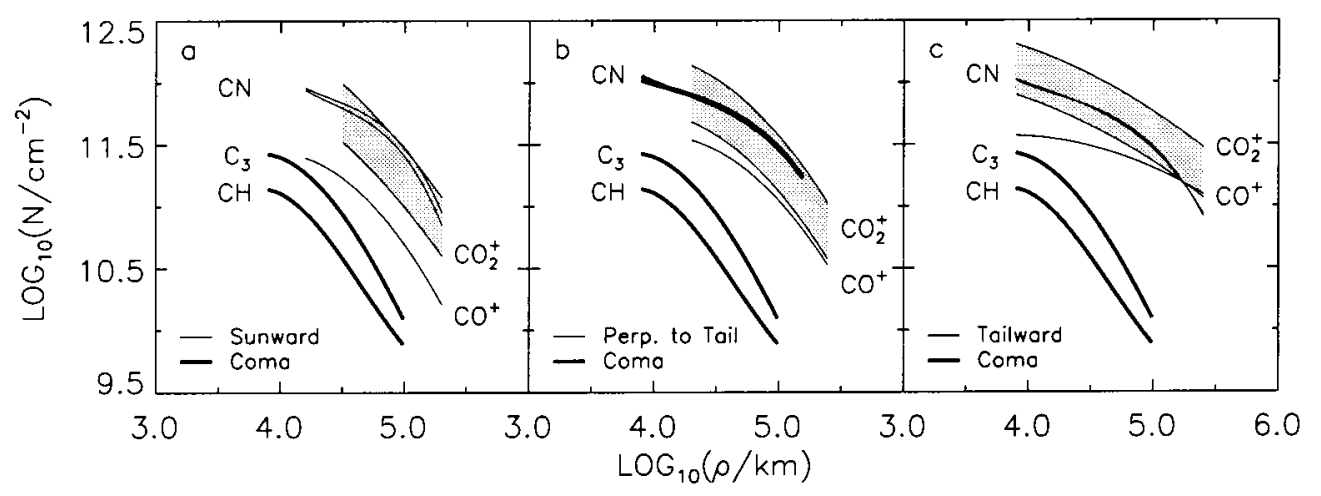

Fig. 7. Radial column density profiles of observed coma constituents a-c) for different areas of the coma

Table 3. Strength of the dust continuum and the averaged CN column densities as a function of the projected distance from nucleus

\begin{tabular}{|c|c|c|c|}
\hline \multirow{2}{*}{$\begin{array}{l}\text { Distance } \\
\log _{10}(\rho) \\
(\mathrm{km})\end{array}$} & \multicolumn{2}{|c|}{ Dust continuum $^{\mathrm{a}}$} & \multirow{2}{*}{$\begin{array}{l}\mathrm{CN} \\
\log _{10}(N) \\
\left(\mathrm{cm}^{-2}\right)\end{array}$} \\
\hline & $\begin{array}{l}\log _{10}(I) \\
\left(I_{\odot}\right)\end{array}$ & $\begin{array}{l}\log _{10}(p \Phi(\alpha) f \rho) \\
(\mathrm{cm})\end{array}$ & \\
\hline 3.9 & -10.72 & 3.09 & 12.01 \\
\hline 4.0 & -10.32 & 3.59 & 12.07 \\
\hline 4.1 & -10.78 & 3.23 & 11.95 \\
\hline 4.2 & -10.79 & 3.32 & 11.92 \\
\hline 4.3 & -10.90 & 3.31 & 11.91 \\
\hline \multicolumn{4}{|l|}{4.4} \\
\hline 4.5 & -10.97 & 3.44 & 11.82 \\
\hline 4.6 & -11.15 & 3.36 & 11.76 \\
\hline 4.7 & -11.35 & 3.26 & 11.66 \\
\hline 4.8 & -11.36 & 3.35 & 11.63 \\
\hline 4.9 & -11.49 & 3.32 & 11.53 \\
\hline 5.0 & -11.64 & 3.27 & 11.47 \\
\hline 5.1 & -11.75 & 3.26 & 11.34 \\
\hline 5.2 & -11.86 & 3.25 & 11.22 \\
\hline 5.3 & -11.91 & 3.30 & 11.12 \\
\hline 5.4 & -11.99 & 3.32 & 10.93 \\
\hline 5.5 & -12.35 & 3.06 & 10.94 \\
\hline
\end{tabular}

a At $3650 \AA$.

The mean solar disc intensity at $3650 \AA$ is $I_{\odot}=$ $1.58310^{6} \mathrm{erg} \mathrm{cm}^{-2} \mathrm{~s}^{-1} \AA^{-1} \mathrm{sr}^{-1}$, i.e. the solar flux after Kurucz et al. (1984) divided by the solid angle subtended by the sun at $1 \mathrm{AU}$ (Unsöld \& Baschek 1988). For comparison of the dust intensities with the literature the following equation (Jockers et al. 1993) was applied:

$p \Phi(\alpha) f \rho=\frac{I}{I_{\odot}}\left(\frac{r}{R_{\odot}}\right)^{2} \rho$,

where $p$ is the geometric albedo, $\alpha$ the phase angle of the comet, and $\Phi(\alpha)$ the phase function. In the present paper the filling factor $f$ is the local filling factor at the projected distance $\rho$ from the cometary optocentre. $p \Phi(\alpha) f \rho$ is expected to be a constant if $I / I_{\odot}$ varies with $\rho^{-1}$. The average of the table entries is $p \Phi(\alpha) f \rho=2.1(6) 10^{3} \mathrm{~cm}$. Note that the Albedo-filling factor-distance product $A f \rho$ used by A'Hearn et al. (1984), and, e.g., Osip et al. (1992) and Storrs et al. (1992) must be divided by 8 in order to be comparable with our value (a factor of 4 arises from the incorrect use of albedo by these authors and a further factor of 2 from the fact that we refer to the local filling factor at the projected distance $\rho$ and not to the filling factor averaged over a circular aperture with radius $\rho$, see Jockers \& Bonev 1997). For example, the value of Af $\rho$ provided by Osip et al. (1992) for comet Halley at the Giotto encounter of $\log (A f \rho)=4.28$ translates to a value of $p \Phi(\alpha) f \rho=2.410^{3} \mathrm{~cm}$.

It is not possible to directly compare our value with the (similar) value of Osip et al. because we observed the comet at larger heliocentric distance and a reduced phase angle as compared to the time of the Giotto encounter. In addition, Comet $\mathrm{P} /$ Halley showed brightness fluctuations with a period of 7.3 days (Neckel \& Münch 1987). Therefore, our data should only be compared with results of publications referring to the same observation time. Neckel \& Münch (1987) have performed aperture photometry of comet Halley. They provide four measurements between April 10.05 and 10.07, 1986, for four different circular apertures centered at the nucleus. One filter (C) used by these authors peaked at $5250 \AA$ with an effective equivalent width of $100 \AA$. This wavelength band excluded significant cometary emission lines and is close to the effective wavelength of the $V$-band of the standard $U B V$ filter system. Neckel \& Münch provide the cometary brightness measured in their continuum filter as $V$ magnitudes in the standard Johnson $U B V$ system. The solar magnitude $m_{V}$ equals -26.74 (Allen 1973). If we transfer fluxes to intensities by making use of the apertures employed by Neckel and Münch (we did not use aperture 2 because it was inconsistent with the other apertures) and 
the angular size of the solar disk, and divide the resulting cometary intensity by two to transfer the apertureaveraged intensity to the local intensity we obtain from Eq. (4) $p \Phi(\alpha) f \rho=1.3010^{3} \mathrm{~cm}$ at $\lambda=5500 \AA$, the effective wavelength of the $V$-band. With Eq. (2) we obtain at $3650 \AA p \Phi(\alpha) f \rho=1.0610^{3} \mathrm{~cm}$. This is about the half of our value. This deviation is qualitatively confirmed by a similar comparison (see below) for $\mathrm{CN}$ column densities.

The profiles of the $\mathrm{CN}$ emissions (Figs. 5d-i) indicate a nearly symmetrical cyanogen distribution. The small deviation between the sunward and tailward profiles arises from the acceleration effect of the solar radiation pressure on these particles. For P/Halley similar observations were made e.g. by Arpigny et al. (1986a) and Ellis \& Neff (1992). Combi \& Delsemme (1980) developed a model to determine the strength of that effect and published computed neutral profiles which are in excellent agreement with our $\mathrm{CN}$ profiles.

The $\mathrm{C}_{3}$ intensity profile (Fig. $5 \mathrm{j}$ ) shows a strong decrease toward the outer coma. The detectable $\mathrm{C}_{3}$ coma is too small to reveal the effect of the radiation pressure. The CH profile (Fig. 5k) also does not show this effect, although Wyckoff et al. (1988) reported a slight asymmetry between the sunward and tailward parts of their $\mathrm{CH}$ profile for $\mathrm{P} /$ Halley.

The ion profiles are plotted in Fig. 6. The vertical extent of the cloud of data in these panels is much larger than for the neutral emissions. This indicates the strong deviation of the plasma cloud from spherical symmetry which is caused by the interaction of the charged particles with the solar wind pushing the ions tailward. The $\mathrm{CO}_{2}^{+}$distribution is displayed in Figs. 6a-f and is in general agreement with the variation of the $\mathrm{CO}_{2}^{+}$emission around $2890 \AA$ that was found in sunward and tailward spectra of comet Bradfield 1979 X (Festou et al. 1982). Both kinds of tailward profiles, the normal one (Figs. 6ac) and that based on consideration of the pseudocontinua $\left({ }^{\mathrm{P}}\right.$, Figs. 6d-f), suggest a local maximum in the ionic coma content at about $\rho=10^{5} \mathrm{~km}$. For the profiles with and without pseudocontinuum the same polynomial fits were used, but with an intensity offset. The profiles of the $\mathrm{CO}^{+}$ bands are shown in Figs. 6g-o. Only the $(2-0)$ band could be used to fit reliable profiles, because the $(3-0)$ and $(4-0)$ intensities near the nucleus are probably still influenced by other emissions. The tailward profiles of $\mathrm{CO}^{+}$ also show the local ion maximum.

Column density profiles. With Eq. (3) the fitted molecular intensity profiles were transformed to column density profiles which are shown in Figs. 7a-c. The profiles of CN $(0-0)$ and $(0-1)$ are in good agreement for the coma and the tailward areas, respectively, only the sunward profiles show a slight offset. The relative error of all CN column densities is found to be not worse than about $10 \%$. The column densities were averaged (Table 3 ) and the resulting mean profile was quantitatively compared with the
CN profile of $\mathrm{P} /$ Halley for April 10.40, 1986, that was published by Combi et al. (1994). Our column densities are larger by a factor of about $10^{0.23} \approx 1.7$ than the referenced profile. This deviation is in qualitative agreement with the analogous factor of 2 which was deduced (see above) from a comparison for dust continuum intensities.

For the $\mathrm{C}_{3}$ and $\mathrm{CH}$ radicals only coma profiles could be fitted. Both profiles show a more rapid decrease with increasing distance from nucleus than in the case of CN. For $\mathrm{C}_{3}$ this is in agreement with Goraya et al. (1988), Hu et al. (1988), and Ellis \& Neff (1992). The $\mathrm{C}_{3}$ profile is only slightly steeper than the $\mathrm{CH}$ profile. Figures $7 \mathrm{a}-\mathrm{c}$ indicate the coma extension at a constant column density: the $\mathrm{CN}$ coma is the largest one followed by the $\mathrm{C}_{3}$ and $\mathrm{CH}$ comae. Mitchell et al. (1981) created a model to predict particle density profiles for several neutral coma constituents. Their calculated abundances for $\mathrm{CN}, \mathrm{C}_{3}$, and $\mathrm{CH}$, are in good agreement with our observed profiles, whereas the gradients of their profiles do not describe the present profiles well.

For $\mathrm{CO}_{2}^{+}$two sets of profiles, connected by a shaded area, are shown in Fig. 7. The upper profiles were directly deduced from the overall intensity in the $\mathrm{CO}_{2}^{+}$integration range, whereas the lower profiles take an additional pseudocontinuum into account. As mentioned above, the two profiles differ only by a constant, which gives credibility to the relative trend. The tailward profile of $\mathrm{CO}_{2}^{+}$ shows a steeper gradient than the corresponding $\mathrm{CO}^{+}$profile; this is consistent with observations of comet West 1976 VI (A'Hearn \& Feldman 1980). The sunward profiles do not reveal significant differences in the decrease of the two species. The profile perpendicular to the tail shows a slightly larger decrease of $\mathrm{CO}_{2}^{+}$as compared to $\mathrm{CO}^{+}$. Wegmann et al. (1987) have developed a threedimensional model to determine particle density profiles for different ionic coma constituents. This model confirms the steeper gradients perpendicular to the tail but in contrast to our observations the tailward gradient of $\mathrm{CO}^{+}$ and $\mathrm{CO}_{2}^{+}$is nearly equal. Because all ions move with the same velocity nearly parallel to the tail axis, the steeper gradient perpendicular to the tail indicates a less extended source region for $\mathrm{CO}_{2}^{+}$. The observed steeper gradient in tailward direction could be caused by a destruction process of $\mathrm{CO}_{2}^{+}$, which is not implemented in the model, like perhaps photodissociation. This additional process would lead to a shorter $\mathrm{CO}_{2}^{+}$mean life time as compared to $\mathrm{CO}^{+}$.

The ion profiles in Fig. 7 indicate, even if a $\mathrm{CO}_{2}^{+}$pseudocontinuum is considered, that $\mathrm{CO}^{+}$is less abundant than $\mathrm{CO}_{2}^{+}$. This conclusion is inconsistent with observations for comet Giacobini-Zinner by A'Hearn et al. (1986) which found a column density for $\mathrm{CO}^{+}(2188 \AA)$ that might be about a magnitude higher than their value for $\mathrm{CO}_{2}^{+}(2888 \AA)$. Supplementary, Krankowsky (1991) reported a $\mathrm{CO}$ production rate for $\mathrm{P} / \mathrm{Halley}$ that is larger than that of $\mathrm{CO}_{2}$. The photochemical lifetime of these neutral molecules, the most important parents for $\mathrm{CO}^{+}$ 
and $\mathrm{CO}_{2}^{+}$, is similar. Therefore their abundance ratio is expected to be roughly transferred by the photoionization process to their daughter molecules. Also the models of Ip (1981) and Wegmann et al. (1987) predict larger particle densities for $\mathrm{CO}^{+}$than for $\mathrm{CO}_{2}^{+}$. The inconsistency between these models and our data may be explained by the performed intensity calibration: in the $\mathrm{CO}_{2}^{+}$wavelength range the response function had to be extrapolated (see Fig. 3b). After all, it is probably necessary to consider a pseudocontinuum for $\mathrm{CO}_{2}^{+}$. Additionally, the $\mathrm{CO}_{2}^{+}$column densities depend on preliminary fluorescence emission rates.

\subsection{Haser scale lengths and production rates}

There is no doubt that the Haser (1957) model is too simple to adequately describe the distribution of neutral radicals in a cometary coma (see Festou et al. 1993, Sect. 4.3.2), but it is still widely used. Therefore, in order to compare our data set with others we have determined Haser scalelengths from our profiles. The model was implemented using equations for the Bessel functions of Abramowitz \& Stegun (1984). For the determination of the parent and daughter scale lengths, $p$ and $d$, their parameter space was searched for those values leading to an absolute minimum of the $\chi^{2}$ function which was defined in the usual way by comparing the normalized observed coma profile with the modeled one. The results are given in Table 4. For $\mathrm{C}_{3}$ and $\mathrm{CH}$, respectively, this method yielded a parameter pair that fits the observed profiles best, whereas the $\mathrm{CN}$ profiles could be fitted with several pairs of scale lengths (see also Cochran 1985). This is caused by the huge extent of the $\mathrm{CN}$ coma compared even with our field of $\approx 0.5^{\circ}$ diameter. Therefore, a nominal value for the CN daughter scale length was taken from A'Hearn (1982). Meredith et al. (1992) present CN parent scales for a number of comets. These authors show that a scaling of the scale length according to the square heliocentric distance (as was done by us) frequently is not a good fit to their data. When this is taken into account our parent scale length agrees well with their values for minimum solar activity conditions (comet Halley).

Table 4. Haser scale lengths and production rates

\begin{tabular}{|c|c|c|c|}
\hline Molecule & $\begin{array}{l}p^{\mathrm{a}} \\
(\mathrm{km})\end{array}$ & $\begin{array}{l}d^{\mathrm{a}} \\
(\mathrm{km})\end{array}$ & $\begin{array}{l}Q / v \\
\left(\mathrm{~km}^{-1}\right)\end{array}$ \\
\hline $\mathrm{CN}(0-0)$ & $2.110^{4}$ & $310^{5 b}$ & $8.2(1) 10^{26}$ \\
\hline$(0-1)$ & $1.910^{4}$ & $310^{5 \mathrm{~b}}$ & $7.7(1) 10^{26}$ \\
\hline $\mathrm{C}_{3}$ & $6.910^{3}$ & $5.910^{4}$ & $5.9(2) 10^{25}$ \\
\hline $\mathrm{CH}$ & $7.510^{3}$ & $6.310^{4}$ & $3.3(2) 10^{25}$ \\
\hline
\end{tabular}

a Reduced to $r=1$ AU with $r^{2}$ scaling law.

b A'Hearn (1982).
Using the obtained scale lengths and the method of Newburn \& Spinrad (1984) the quantity $Q / v$, i.e. the production rate divided by the neutral radial outflow velocity, was determined for each radical. Assuming $v=1 \mathrm{~km} \mathrm{~s}^{-1}$ $Q / v$ can easily be transformed to a production rate. The given rmse represents the goodness of the fit.

\section{Maps}

Intensity and column density maps. The distributions of the dust continuum and the molecular species are shown as contour plots in Figs. 8 and 9. These two-dimensional maps present the measured continuum intensities and column densities in logarithmic scale with respect to their spatial resolution (compare Fig. 1). Generally, the distributions decrease from a peak near the cometary nucleus $(+)$ towards the outer coma. In the maps the shape of the contour lines strongly depends on single values of the nonuniformly distributed data points (intensity or column density values and their corresponding slit positions). Therefore, some strongly deviating data points could not be used for properly interpolating the maps. In the case of the data sets $\mathrm{A}$ and $\mathrm{C}$ the cometary nucleus is covered quite well with a slit column of the slit plate (Fig. 1), and therefore, the centers of the interpolated distributions are very close to the nucleus. For data set $B$ this condition is not met, and the centers of the deduced distributions differ from the position of the nucleus. The neutral distributions do not show a variation with time. Therefore, the three corresponding maps of each considered neutral coma constituent were superposed to create additional maps with a higher spatial resolution (Figs. 8p-t).

The dust coma has a nearly symmetrical distribution within a projected radius of approximately $10^{5} \mathrm{~km}$ which is best seen in the superposed dust map. The detectable dust coma was well covered by the large field of view of the focal reducer, but only the innermost part of the dust tail, that had a length of several degrees at observation time (Ozeki 1986; Lamy et al. 1987), appears in the lower left part of the maps.

As expected from the CN profiles, the cyanogen maps also reveal quite a symmetrical $\mathrm{CN}$ coma with a slight deformation caused by the effect of the solar radiation pressure (Sect. 5.1). The contour lines of the CN $(0-1)$ maps are not as circular as those of the $\mathrm{CN}(0-0)$ maps because the related band intensities are smaller and have a larger relative error, but the general agreement is very good. Kidger et al. (1987) also published maps for both $\mathrm{CN}$ emissions. The $\mathrm{C}_{3}$ coma is found to have a symmetrical particle distribution. Kidger et al. (1987) also found no significant deviation from spherical symmetry. In our maps, the shape of the contour lines in the lower left part may be due to an unidentified ion emission contaminating the $\mathrm{C}_{3}$ emission. The $\mathrm{CH}$ maps of the three data sets are not very similar. Therefore, the strength of the $\mathrm{CH}$ emission mark a lower intensity limit: weaker emissions 


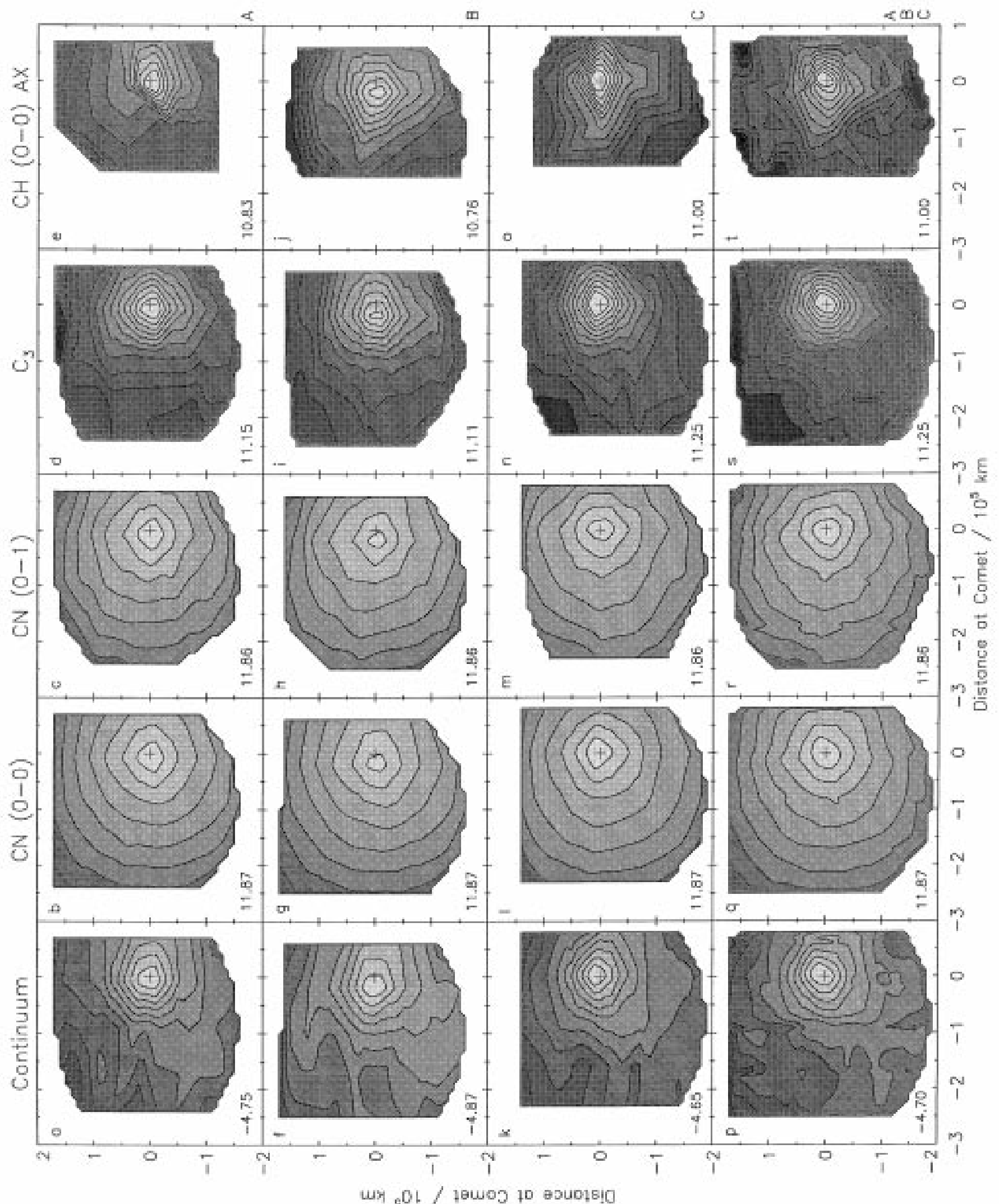

Fig. 8. Celestial plane projection of the distribution of neutral coma constituents for the data sets A (a-e), B (f-j), C (k-o), and their superposition ABC (p-t). The values of the innermost contour lines are given in $\log _{10}\left(I / \mathrm{erg} \mathrm{cm}^{-2} \mathrm{~s}^{-1} \AA^{-1} \mathrm{sr}^{-1}\right)$ in the case of the dust continuum at $3650 \AA$, and in $\log _{10}\left(\mathrm{~N} / \mathrm{cm}^{-2}\right)$ in the case of the radicals; the contour level decrement is always $\frac{1}{2} \log _{10}(2)=0.1505$ 


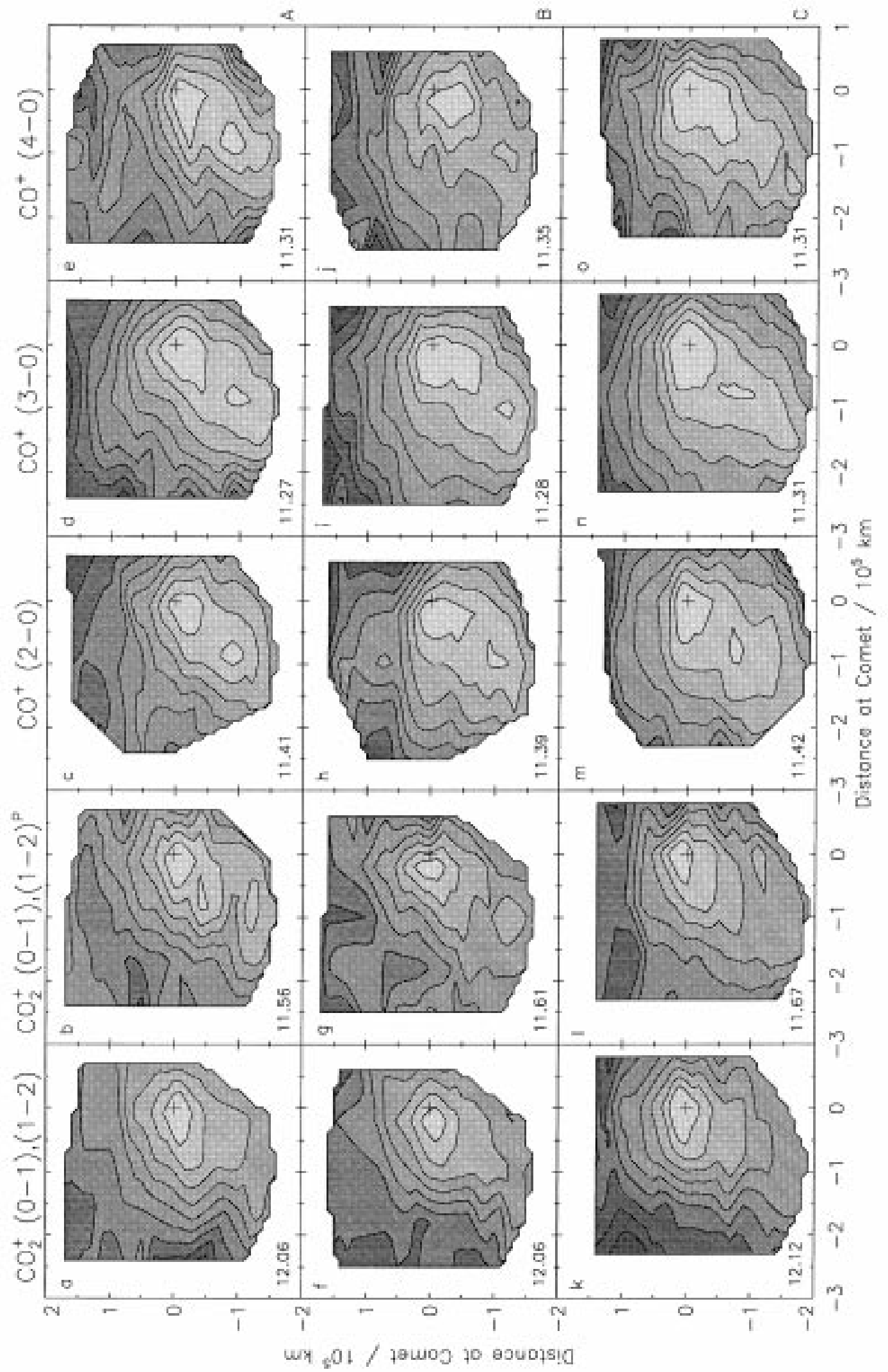

Fig. 9. Celestial plane projection of the distribution of ionic coma constituents for the data sets A (a-e), B (f-j), and C (k-o). The values of the innermost contour lines are given in $\log _{10}\left(\mathrm{~N} / \mathrm{cm}^{-2}\right)$; the contour level decrement is equal to 0.1505 
could not be used for interpolating reliable profiles and maps. The $\mathrm{CH}$ emission in the spectra of the left slit column were too weak to be measured. The superposed $\mathrm{CH}$ map reveals a detectable $\mathrm{CH}$ coma that is slightly smaller than the $\mathrm{C}_{3}$ coma. The presented maps for $\mathrm{CN}(0-0)$, $\mathrm{C}_{3}$, and $\mathrm{CH}$, are in agreement with the observations by Kidger et al. (1987).

The distributions of $\mathrm{CO}^{+}$and $\mathrm{CO}_{2}^{+}$show a significant deviation from spherical symmetry that is caused by the interaction of these particles with the solar wind. The decrease towards the sun is always stronger than that in tailward direction, and the tailward gradient of $\mathrm{CO}^{+}$is found to be less steep than that of $\mathrm{CO}_{2}^{+}$. Close to the nucleus, the ion maps are much flatter than the maps of the neutral species and the dust, and show a plateau extending from the nucleus into the tail. A similar flat distribution of the $\mathrm{H}_{2} \mathrm{O}^{+}$ion has been observed in comet Austin by Bonev \& Jockers (1994). The ion maps which are based on an intensity integration that considered a pseudocontinuum (Figs. 9b-e, g-j, l-o) suggest the existence of plasma clouds in the tail. This is supported by Jockers et al. (1987) which published a time series of direct filter images of comet $\mathrm{P} /$ Halley for April 11, 1986, in the light of $\mathrm{CO}_{2}^{+}$ $(3674 \AA)$ and $\mathrm{CO}^{+}(4252 \AA)$ that both show the ejection of a plasma cloud. The modeled column density maps for $\mathrm{CO}_{2}^{+}$and $\mathrm{CO}^{+}$by Wegmann et al. (1987) are consistent with our large scale distributions with the exception of the tailward gradient observed for $\mathrm{CO}_{2}^{+}$.

\section{Conclusions}

For different coma areas representative spectra were presented in the wavelength range from $3600-4400 \AA$. They allow an easy comparison between the emissions of the cometary head and tail. The spectra were obtained with the multislit technique, which allowed to record 69 spectra of different coma regions at the same time. The head spectra are dominated by emissions of the neutral radicals $\mathrm{CN}, \mathrm{C}_{3}$, and $\mathrm{CH}$, whereas the emissions of $\mathrm{CO}^{+}$and $\mathrm{CO}_{2}^{+}$ most importantly contribute to the tail spectra. The ion emissions are not restricted to a sharp plasma tail, but are also present in the sunward coma.

The results about the distribution of dust and neutral molecules in the cometary coma mostly agree with the literature. The radial profile of the overall dust coma is consistent with the $\rho^{-1}$ law expected from the radial outflow model. The continuum strength is about twice as large as expected from data published by Neckel \& Münch (1987). The CN profiles clearly show the influence of the solar radiation pressure on neutral particles as modeled by Combi \& Delsemme (1980). The CN data indicate a relative error of about $10 \%$, and the $\mathrm{CN}$ column densities are larger than those of Combi et al. (1994) by a factor of 1.7. The deviations of the dust continuum and the CN column densities indicate that our values should be regarded as upper limits for the abundance of the considered species. The neutral coma model of Mitchell et al. (1981) is consistent with the relative abundances of $\mathrm{CN}, \mathrm{C}_{3}$, and $\mathrm{CH}$, but not with the gradients of these distributions. Concerning the ions we present the first observation of the two-dimensional spatial distribution of the $\mathrm{CO}_{2}^{+}$ion. The observations are in agreement with the model of Wegmann et al. (1987) with the exception of a stronger tailward gradient of the ion as compared to the model. Our $\mathrm{CO}_{2}^{+}$abundance is too high which may be due to observational difficulties or to problems with the (preliminary) fluorescence emission rate.

The method of multislit spectroscopy in combination with a focal reducer is very well suited to obtain a large number of spectra of an object as extended as a comet. The most important cometary emissions within a wide spectral range are simultaneously observable and their large-scale spatial distribution can be determined.

Acknowledgements. The authors would like to thank S.J. Kim from the Department of Astronomy and Space Science of the Kyunghee University, Yongin, Kyunggido, South Korea, for providing fluorescence emission rates for $\mathrm{CO}_{2}^{+}$. We also like to express our thanks to M. Richards from the Max-PlanckInstitut für Aeronomie for discussions and for placing data reduction software at our disposal that was developed by him. We are grateful for the technical support of P. Grosbøl from ESO Headquarters while scanning the plates.

\section{References}

Abramowitz M., Stegun I.A., 1984, Pocketbook of mathematical functions. Harri Deutsch, Thun

A'Hearn M.F., 1982, in: Comets, Wilkening L.L. (ed.). University of Arizona Press, Tucson, AZ, p. 443

A'Hearn M.F., Feldman P.D., 1980, ApJ 242, L187

A'Hearn M.F., Schleicher D.G., Feldman P.D., Millis R.L., Thompson D.T., 1984, AJ 89, 579

A'Hearn M.F., McFadden L.A., Feldman P.D., Böhnhard H., Rahe J., Festou M., Brandt J.C., Maran S.P., Niedner M.B., Smith A.M., Schleicher D.G., 1986, in: New insights in astrophysics, Rolfe E.J. (ed.), ESA SP-263, p. 35

Allen C.W., 1973, Astrophysical quantities. Athlone Press, London

Aller L.H., Czyzak S.J., 1978, Proc. Natl. Acad. Sci. U.S.A. 75,1

Aller L.H., Ross J.E., O’Mara B.J., Keyes C.D., 1981, MNRAS 197, 95

Arpigny C., Dossin F., Manfroid J., Magain P., Danks A.C., Lambert D.L., Sterken C., 1986a, ESO Messenger 45, 8

Arpigny C., Dossin F., Zucconi J.M., Andrillat Y., Manfroid J., Hutsemékers D., 1986b, in: Exploration of Halley's comet, Battrick B., Rolfe E.J., Reinhard R. (eds.), ESA SP-250 Vol. 1, p. 471

Brocklehurst B., Hébert G.R., Innanen S.H., Seel R.M., Nicholls R.W., 1972, Identification atlas of molecular spectra, 9. The CN $B^{2} \Sigma^{+}-X^{2} \Sigma^{+}$violet system, York University, Toronto

Bonev T., Jockers K., 1994, Icarus 107, 335

Clairemidi J., Moreels G., Krasnopolsky V.A., 1990, Icarus 86, 115

Cochran A.L., 1985, AJ 90, 2609 
Combi M.R., Delsemme A.H., 1980, ApJ 237, 633

Combi M.R., Fink U., 1993, ApJ 409, 790

Combi M., Huang B., Cochran A., Fink U., Schulz R., 1994, ApJ 435, 870

Cromwell R.H., Smith G.H., 1973, Proc. SPIE 42, 155

Cromwell R.H., Strittmatter P.A., Allen R.G., Hege E.K., Kühr H., Marien K.-H., Funk H.W., Frank K., 1985, Adv. E.E. P. 64A, 77

Danks A.C., 1983, The ESO user's manual, European Southern Observatory. Garching

Danziger I.J., Frogel J.A., Persson S.E., 1973, ApJ 184, L29

Eccles M.J., Sim M.E., Tritton K.P., 1983, Low light level detectors in astronomy. Cambridge University, Cambridge

Ellis T.A., Neff J.S., 1992, Icarus 97, 99

Festou M.C., Feldman P.D., Weaver H.A., 1982, ApJ 256, 331

Festou M.C., Rickman H., West R.M., 1993, A\&A Rev. 5, 37

Gammelgaard P., Thomson B., 1988, A\&A 197, 320

Gausset L., Herzberg G., Lagerqvist A., Rosen B., 1965, ApJ 142,45

Gerö L., 1941, Z. Physik 118, 27

Geyer E.H., Hoffmann M., Nelles B., 1979, A\&A 80, 248

Goraya P.S., Sanwal B.B., Rautela B.S., 1988, Earth Moon Planets 42, 113

Green D.W.E., 1985, IAU Circ. 4156

Haser L., 1957, Bull. Acad. Roy. Belg. (Classe Sci.) 43, 740

Hu Z., Shen Z., Chen Y., 1988, Acta Astron. Sin. 29, 129

Ip W.-H., 1981, in: Scientific and experimental aspects of the Giotto mission, Battrick B., Mort J. (eds.), ESA SP-169, p. 79

Jewitt D.C., Meech K.J., 1987, ApJ 317, 992

Jockers K., Bonev T., 1997, A\&A 319, 617

Jockers K., Geyer E.H., Rosenbauer H., Hänel A., 1987, A\&A 187,256

Jockers K., Kiselev N.N., Boehnhardt H., Thomas N., 1993, A\&A 268, L9

Kidger M.R., Acosta J.A., Garzón F., Prieto M., Gómez R., 1987, A\&A 167, 363

Krankowsky D., 1991, in: Comets in the post-Halley era, Vol. 2, Newburn R.L., Neugebauer M., Rahe J. (eds.). Kluwer Academic Publishers, Dordrecht, p. 855

Kurucz R.L., Furenlid I., Brault J., Tester L., 1984, Solar flux atlas from 296 to $1300 \mathrm{~nm}$. Harvard University Press,
Cambridge, MA

Lamy P.L., Pedersen H., Vio R., 1987, A\&A 187, 661

Levasseur-Regourd A.C., Bertaux J.L., Dumont R., Festou M., Giese R.H., Giovane F., Lamy P., Le Blanc J.M., Llebaria A., Weinberg J.L., 1986, Nat 321, 341

Loewen E.G., 1970, Diffraction grating handbook, Bausch \& Lomb Diffraction Grating Res. Lab., Rochester, NY

Lutz B.L., Womack M., Wagner R.M., 1993, ApJ 407, 402

Magnani L., A'Hearn M.F., 1986, ApJ 302, 477

McCallum J.C., Nicholls R.W., 1972, J. Phys. B: Atom. Molec. Phys. 5, 1417

Meech K.J., Jewitt D.C., 1987, A\&A 187, 585

Meredith N.P., Wallis M.K., Rees D., 1992, MNRAS 254, 693

Mitchell G.F., Prasad S.S., Huntress W.T., 1981, ApJ 244, 1087

Mrozowski S., 1947a, Phys. Rev. 72, 682

Mrozowski S., 1947b, Phys. Rev. 72, 691

Neckel T., Münch G., 1987, A\&A 187, 581

Newburn R.L., Spinrad H., 1984, AJ 89, 289

Oliver J.P., Aller L.H., 1969, ApJ 157, 601

Osip D.J., Schleicher, D.G., Millis, R.L., 1992, Icarus 98, 115

Ozeki T., 1986, IHW Newslett. 8, 4

Rousselot P., Clairemidi J., Moreels G., 1994, A\&A 286, 645

Storrs A.D., Cochran A.L., Barker E.S., 1992, Icarus 98, 163

Swings P., Haser L., 1956, Atlas of representative cometary spectra, University of Liège, Liège

Tatum J.B., 1984, A\&A 135, 183

Thomas, N., Keller H.U., 1989, A\&A 213, 487

Unsöld A., Baschek B., 1988, Der neue Kosmos. Springer, Berlin

Valk J.H., O'Dell C.R., Cochran A.L., Cochran W.D., Opal C.B., Barker E.S., 1992, ApJ 388, 621

Wegmann R., Schmidt H.U., Huebner W.F., Boice D.C., 1987, A\&A 187, 339

Werner, R., Guineva, V., Stoeva, P., Spasov, S., 1989, Adv. Space Res. 9, 221

West R.M., 1988, ESO scientific preprint 624, European Southern Observatory. Garching

Wyckoff S., Tegler S., Wehinger P.A., Spinrad H., Belton M.J.S., 1988, ApJ 325, 927

Wyckoff S., Theobald J., 1989, Adv. Space Res. 9, 157

Zucconi J.M., Festou M.C., 1985, A\&A 150, 180 